\title{
Optimal behavior of the support of the solutions to a class of degenerate parabolic systems
}

\author{
ANATOLI TEDEEV \\ South Mathematical Institute of Vladikavkaz Scientific Center of the Russian Academy of Sciences, \\ Markus str. 22, 362027 Vladikavkaz, Russia \\ E-mail:a_tedeev@yahoo.com \\ VINCENZO VESPRI \\ Dipartimento di Matematica ed Informatica Ulisse Dini, Università degli studi di Firenze, \\ Viale Morgagni, 67/a, 50134 Firenze, Italy \\ E-mail:vincenzo.vespri@unifi.it
}

[Received 26 January 2014 and in revised form 16 February 2015]

\begin{abstract}
In this paper we deal with a class of degenerate/singular quasilinear parabolic systems. We study a Cauchy problem in $\mathbb{R}^{N}$ with an initial datum in $L^{1}$. Sharp $L^{\infty}$ estimates are proved. In the degenerate case, assuming that the initial datum has compact support, we prove the optimal speed of propagation of the support.
\end{abstract}

2010 Mathematics Subject Classification: Primary 35K59; Secondary 35K92, 35K45, 35B65

Keywords: Degenerate and singular parabolic systems, $L^{\infty}$ estimates, finite speed of the propagation of the support

\section{Introduction}

In this paper we consider the following Cauchy problem, where $j=1, \ldots, l$

$$
\left\{\begin{array}{l}
\frac{\partial u_{j}}{\partial t}=\sum_{i=1}^{N} \frac{\partial}{\partial x_{i}}\left(|\mathbf{U}|^{m-1}|\nabla \mathbf{U}|^{p-2} \frac{\partial}{\partial x_{i}} u_{j}\right), \quad \text { in } S_{T}=\mathbb{R}^{N} \times(0, T), \\
u_{j}(x, 0)=u_{0 j}(x),
\end{array}\right.
$$

where here and hereafter bold letters are standing for vectors of length $l$ :

$$
\mathbf{U}=\left(u_{1}, \ldots, u_{l}\right), \quad l \geqslant 1, \quad|\nabla \mathbf{U}|:=\left(\sum_{i=1}^{l}\left|\nabla u_{i}\right|^{2}\right)^{1 / 2} .
$$

Such kind of systems are named doubly nonlinear. The doubly nonlinear equations were introduced by Lions ([19]) and Kalashnikov ([15]) several decades ago. These equations, from one side, are used to model several physical phenomena, on the other side, they are a natural bridge between two of the main important quasilinear equations, i.e., porous medium and $p$-Laplacean (for references on these protype equations, see for instance [23]. For these reasons many Authors studied these equations. Among them, we quote Tsustumi ([22]), Ivanov ([13, 14]), Porzio and Vespri ([20]), 
Ishige ([12]) and Fornaro and Sosio ([11]). In these papers the existence, the Hölder regularity and intrinsic Harnack properties of these solutions are proved. For further results concerning the qualitative properties of solutions of degenerate parabolic equation we refer the reader to the survey ([15]) and to the exhaustive monographs ([8]) and ([23]).

Only recently it was discovered the importance of doubly nonlinear systems in modelling some physical phenomena (see, for instance, [25-28] and [29]). In [5], [6] and [16] the Authors use doubly nonlinear systems to describe the evolution of a fluid in non-Newtonian filtration of the water flow through the porous media, to describe non-equilibrium thermodynamics and to model semiconductors. In [28] (see also [26] and [27]) it was shown that the system of porous medium equations (i.e., $p=2$ in (1)) comes from Bean's critical-state model in the superconductivity theory.

For systems (1) with $m=1$ and with more general structure let us quote [9] for the $C^{1, \alpha}$ regularity of the solutions.

To our knowledge, regularity estimates for solutions of the Cauchy problem (1) (and even for systems with more general structure) are proved only in [17]. In this paper the Authors prove sharp $L^{1}-L^{\alpha}$ estimates for solutions of (1), provided $u_{i}(x, t) \geqslant 0, i=1, \ldots, l$. Since there is no maximum principle for (1), it is not clear whether the nonnegativity assumption in latter case can be satisfied.

In this paper we drop this not-so-easily verifiable assumption and we begin a systematic study of regularity properties of the weak solutions of (1). More precisely, we focus our attention on the behaviour of the support of the solution in the degenerate case, i.e., when

$$
\left\{\begin{array}{l}
p>1 \\
p+m>3
\end{array}\right.
$$

We prove that the finite speed of propagation of the support occurs exactly as in the case of the corresponding degenerate equations. More precisely, by adapting techniques introduced by Andreucci and Tedeev (see [2], [3] and [4]), we are able to give an optimal estimate of the speed of propagation of the support, due to sharp $L^{\infty}$ estimates. We recall that in ([6], p.179180) Antontsev, Diaz and Shmarev proved the optimal rate of expansion of supports for solutions of systems of degenerate parabolic equations with double nonlinearity, even though the class of systems considered is not the same as here. The methods employed there are different from the ones of this paper, even if both methods start from the energy estimates satisfied by the solutions.

If we limit ourselves to $L^{\infty}$ estimates, we are able to prove them under more general conditions on $m$ and $p$, i.e when

$$
\left\{\begin{array}{l}
p>1 \\
N(p+m-3)+p>0
\end{array}\right.
$$

We recall that in literature the case $m+p<3$ is called singular. When $m+p<3$ and $N(p+m-$ $3)+p>0$ we are in the supercritical range, whereas the case $m+p>2$ and $N(p+m-3)+p \leqslant 0$ is called subcritical (see, for instance, [24] and [10] for such a classification in the case of equations). The case $m+p=3$ was first considered by Trudinger ([21]) and for this reason, such equations are often called Trudinger's equations.

Before stating our main results, let us first define the notion of weak solution of (1). 
A function $\mathbf{U}: S_{T} \rightarrow \mathbb{R}^{l}$ is a weak solution of the Cauchy problem (1)-(3) if

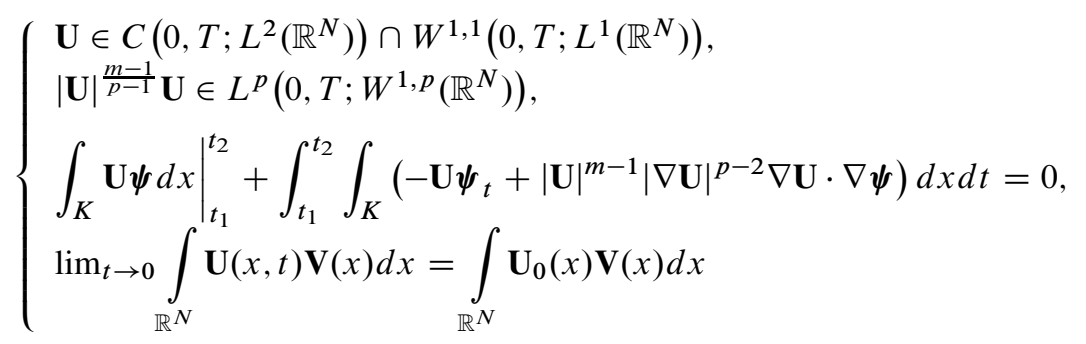

for every compact set $K \subset \mathbb{R}^{N}$, for every sub-interval $\left[t_{1}, t_{2}\right] \subset(0, T]$ and for every test functions

$$
\begin{gathered}
\psi \in W^{1,2}\left(0, T ; L^{2}(K)\right) \cap L^{p}\left(0, T ; W_{0}^{1, p}(K)\right) . \\
\mathbf{V}(x)=\left(v_{1}(x), \ldots, v_{l}(x)\right) \in C_{0}^{1}\left(\mathbb{R}^{N}\right) .
\end{gathered}
$$

The integrability hypothesis on $\mathbf{U}$ ensures that the integrals in (4) are well defined.

Note that in the definition of weak solution we avoid using the time derivative of $\mathbf{U}$, since $\mathbf{U}_{t}$ might not exist as a function. Nevertheless, later on we shall use

$$
\int_{t_{1}}^{t_{2}} \int_{\mathbb{R}^{N}}\left[\mathbf{U}_{t} \boldsymbol{\psi}+|\mathbf{U}|^{m-1}|\nabla \mathbf{U}|^{p-2} \nabla \mathbf{U} \cdot \nabla \boldsymbol{\psi}\right] d x d t=0
$$

instead of (4), as weak form of the equation. Thereby, the use of the time derivative has to be understood in a formal way. These computations can be made rigorous by the use of a mollification procedure with respect to time as Steklov averages, for instance.

THEOREM 1 Let $\mathbf{U}$ be a weak solution of (1) in $S_{\infty}$. Assume that (3) holds and $\mathbf{U}_{0} \in L^{1}\left(\mathbb{R}^{N}\right)$. Moreover, assume that this solution can be approximated by regular problems. Then for any $t>0$

$$
\|\mathbf{U}(t)\|_{\infty} \leqslant C(N, l, m) t^{-\frac{N}{\lambda}}\left\|\mathbf{U}_{0}\right\|_{1}^{\frac{p}{\lambda}},
$$

where $\lambda=N(p+m-3)+p$ is the so-called Barenblatt exponent.

Note that this result is sharp even in the case of equations. Some explicit counterexamples are known in the literature (see, for instance, Chapter 5, Section 4 (iii) of [8] and [23]), where it is shown that estimate (6) fails, whenever $\lambda \leqslant 0$.

As already written, the main result regards optimal estimates about the speed of propagation of the support of the solution:

THEOREM 2 Let $\mathbf{U}$ be a weak solution of (1) in $S_{\infty}$ and supp $\mathbf{U}_{0} \subset B_{R_{0}}(0)=\left\{|x|<R_{0}\right\}$. Assume that (2) holds and $\mathbf{U}_{0} \in L^{1}\left(\mathbb{R}^{N}\right)$. Moreover, assume that this solution can be approximated by regular problems. Then for any $t>0$

$$
\begin{aligned}
Z(t) & =\inf \left\{r>0:|\mathbf{U}(t)|=0, x \in \mathbb{R}^{N} \backslash B_{r}(0)\right\} \\
& \leqslant 4 R_{0}+C(N, l, m) t^{\frac{1}{\lambda}}\left\|\mathbf{U}_{0}\right\|_{1}^{\frac{p+m-3}{\lambda}} .
\end{aligned}
$$

Note that this result is sharp also in the case of simple equations. In fact the support of the Barenblatt solutions exhibits exactly this behaviour (see, for instance, Chapter 5, Section 4 of [8] and [23]). 


\subsection{Remark on the existence of a solution}

In Theorems 1 and 2, we assume the existence of the weak solution of (1) with initial datum in $L^{1}\left(\mathbb{R}^{N}\right)$. We recall that the existence of a weak solution with initial datum in $L^{2}\left(\mathbb{R}^{N}\right)$ can be proved arguing as in [1] (see also [7]). By using the a priori $L^{1}$ estimates proved in this paper and by energy and entropy estimates, it is possible to prove the existence of a solution with an initial datum in $L^{1}$. As the proof is not straightforward and quite long, we will give a detailed argument in a forthcoming paper.

By using the results of this paper, it is possible to weaken the approximation hypotheses. This issue will be considered in the above-mentioned forthcoming paper as well.

\section{$1.2 \quad$ Further generalizations}

Our results can be extended to parabolic systems with measurable coefficients of the form:

$$
\frac{\partial u_{j}}{\partial t}=\sum_{k, \mu=1}^{N} \frac{\partial}{\partial x_{k}}\left(a_{k \mu}(x, t)|\mathbf{U}|^{m-1}|\nabla \mathbf{U}|^{p-2} \frac{\partial}{\partial x_{\mu}} u_{j}\right), \quad j=1, \ldots, l
$$

where $a_{k \mu}(x, t)$ are measurable functions satisfying the conditions

$$
\Lambda^{-1} \xi^{2} \leqslant a_{k \mu}(x, t) \xi_{k} \xi_{\mu} \leqslant \Lambda \xi^{2}, \quad \Lambda \geqslant 1
$$

\section{Preliminary lemmata}

The proof of Theorem 1 is based on some preliminary lemmata.

LEMMA 1 Let $\mathbf{U} \in W^{1,2}\left(\mathbb{R}^{N}\right)$ than for any $\varepsilon \geqslant 0$

$$
\sum_{j=1}^{l} \sum_{k=1}^{N} u_{j x_{k}} \frac{\partial}{\partial x_{k}}\left(\frac{u_{j}}{\left(\varepsilon^{2}+|\mathbf{U}|^{2}\right)^{1 / 2}}\right) \geqslant 0
$$

Proof. Let us develop the calculations:

$$
\frac{\partial}{\partial x_{k}}\left(\frac{u_{j}}{\left(\varepsilon^{2}+|\mathbf{U}|^{2}\right)^{1 / 2}}\right)=\frac{u_{j x_{k}}\left(\varepsilon^{2}+|\mathbf{U}|^{2}\right)-\sum_{s=1}^{l} u_{s} u_{s x_{k}} u_{j}}{\left(\varepsilon^{2}+|\mathbf{U}|^{2}\right)^{3 / 2}} .
$$

Therefore, we have that

$$
\begin{aligned}
\sum_{j=1}^{l} \sum_{k=1}^{N} u_{j x_{k}} & \frac{\partial}{\partial x_{k}}\left(\frac{u_{j}}{\left(\varepsilon^{2}+|\mathbf{U}|^{2}\right)^{1 / 2}}\right) \\
= & \frac{\sum_{j=1}^{l} \sum_{k=1}^{N} u_{j x_{k}}^{2}\left(\varepsilon^{2}+|\mathbf{U}|^{2}\right)-\frac{1}{4} \sum_{k=1}^{N}\left(\sum_{j=1}^{l}\left(u_{j}^{2}\right)_{x_{k}}\right)^{2}}{\left(\varepsilon^{2}+|\mathbf{U}|^{2}\right)^{3 / 2}}
\end{aligned}
$$

Now

$$
\sum_{k=1}^{N} \sum_{j=1}^{l} u_{j x_{k}}^{2}\left(\varepsilon^{2}+|\mathbf{U}|^{2}\right)>\sum_{k=1}^{N} \sum_{j=1}^{l} u_{j x_{k}}^{2}|\mathbf{U}|^{2}
$$


and estimate (10) comes from the fact that by Cauchy-Schwartz inequality

$$
\sum_{j=1}^{l} u_{j x_{k}}^{2}|\mathbf{U}|^{2} \geqslant\left(\sum_{j=1}^{l} u_{j x_{k}} u_{j}\right)^{2}
$$

The next Lemma says that the $L^{1}$ norm of the solution is bounded.

LEMma 2 Let $\mathbf{U}(x, t)$ be a solution of equation (1), then for a.e. $t>0$

$$
\int_{\mathbb{R}^{N}}|\mathbf{U}(x, t)| d x \leqslant \int_{\mathbb{R}^{N}}\left|\mathbf{U}_{0}(x)\right| d x .
$$

Proof. Consider the family of solutions

$$
\left\{\begin{array}{l}
\frac{\partial u_{i}^{(n)}}{\partial t}=\operatorname{div}\left(\left|\mathbf{U}^{(n)}\right|^{m-1}\left|\nabla \mathbf{U}^{(n)}\right|^{p-2} \nabla u_{i}^{(n)}\right) \text { in } B_{n}(0) \times(0, \infty), \\
u_{i}^{(n)}=0 \text { on } \partial B_{n}(0) \times(0, \infty), \\
u_{i}^{(n)}(x, 0)=u_{0 i}^{(n)}(x),
\end{array}\right.
$$

$i=1, \ldots, l$ with $\mathbf{U}_{0}^{(n)}=\left(u_{01}^{(n)}(x), \ldots, u_{0 l}^{(n)}(x)\right)$ smooth enough and such that $\left\|\mathbf{U}_{0}^{(n)}-\mathbf{U}_{0}\right\|_{L^{1}\left(\mathbb{R}^{N}\right)}$ $\rightarrow 0$ as $n \rightarrow \infty$. Then, by assuming that the solution of (1) is the weak limit of $\mathbf{U}^{(n)}$, it is enough to prove that for any $n \geqslant 1$ and $\varepsilon^{-\sigma}$ with $\sigma \geqslant N$

$$
\int_{B_{n}(0)}\left(\varepsilon^{2}+\left|\mathbf{U}^{(n)}(x, t)\right|^{2}\right)^{1 / 2} d x \leqslant \int_{B_{n}(0)}\left(\varepsilon^{2}+\left|\mathbf{U}_{0}^{(n)}(x)\right|^{2}\right)^{1 / 2} d x
$$

for any $t>0$. Multiply both sides of (12) by

$$
\frac{u_{i}^{(n)}}{\left(\varepsilon^{2}+\left|\mathbf{U}^{(n)}\right|^{2}\right)^{1 / 2}}
$$

integrate by parts over $B_{n}(0)$ and add up with respect the index $i$, to get

$$
\begin{aligned}
& \frac{d}{d t} \int_{B_{n}(0)}\left(\varepsilon^{2}+\left|\mathbf{U}^{(n)}\right|^{2}\right)^{1 / 2} d x \\
& \quad=-\int_{B_{n}(0)} \sum_{j=1}^{l} \sum_{k=1}^{N}\left(\left|\mathbf{U}^{(n)}\right|^{m-1}\left|\nabla \mathbf{U}^{(n)}\right|^{p-2} \nabla u_{j x_{k}}^{(n)}\right) \nabla\left(u_{j x_{k}}^{(n)}\left(\varepsilon^{2}+\left|\mathbf{U}^{(n)}\right|^{2}\right)^{-1 / 2}\right) d x
\end{aligned}
$$

By (10) we get

$$
\frac{d}{d t} \int_{B_{n}(0)}\left(\varepsilon^{2}+\left|\mathbf{U}^{(n)}\right|^{2}\right)^{1 / 2} d x \leqslant 0
$$


REMARK 1 Let us sketch the proof of Lemma 2 in the general case. We claim that

$$
a_{k \mu}(x, t) \frac{\partial}{\partial x_{\mu}} u_{j} \frac{\partial}{\partial x_{k}}\left(\frac{u_{j}}{\left(\varepsilon^{2}+|\mathbf{U}|^{2}\right)^{1 / 2}}\right) \geqslant 0 .
$$

Indeed, the left hand side of this inequality is equal to

$$
\frac{a_{k \mu}(x, t) \frac{\partial}{\partial x_{\mu}} u_{j} \frac{\partial}{\partial x_{k}} u_{j}\left(\left(\varepsilon^{2}+|\mathbf{U}|^{2}\right)-u_{j} u_{\alpha} \frac{\partial u_{\alpha}}{\partial x_{k}}\right)}{\left(\varepsilon^{2}+|\mathbf{U}|^{2}\right)^{3 / 2}} .
$$

Next, using the Cauchy inequality for the positive defined quadratic forms

$$
a_{k \mu} \zeta_{k} \eta_{\mu} \leqslant\left(a_{k \mu} \zeta_{k} \zeta_{\mu}\right)^{1 / 2}\left(a_{k \mu} \eta_{k} \eta_{\mu}\right)^{1 / 2}
$$

and choosing

we get the result.

$$
\zeta_{k}=\frac{u_{j}}{|\mathbf{U}|} u_{j x_{k}}, \quad \eta_{\mu}=\frac{u_{\alpha}}{|\mathbf{U}|} u_{\alpha x_{\mu}}
$$

Define $C\left(h_{1}, h_{2}\right)=h_{1} /\left(h_{1}-h_{2}\right)$.

LEMma 3 Assume that the assumptions of Theorem 1 hold. Define $D_{j}=\mathbb{R}^{N} \times\left(t_{j}, t\right), j=1,2$, $0<t_{2}<t_{1}<t$. Then, for all $h_{1}>h_{2}>0$ we have for any $s>0$ so large that $s-2+m-p>0$

$$
\begin{array}{r}
\sup _{t_{1}<\tau<t} \int_{\mathbb{R}^{N}}\left(|\mathbf{U}|^{2}-h_{1}\right)_{+}^{(s+1) / 2} d x+\iint_{D_{1}}\left(|\mathbf{U}|^{2}-h_{1}\right)_{+}^{\frac{s+m-2-p}{2}}\left|\nabla\left(|\mathbf{U}|^{2}-h_{1}\right)_{+}\right|^{p} d x d \tau \\
\leqslant \gamma C\left(h_{1}, h_{2}\right)^{p+m-1}\left(t_{1}-t_{2}\right)^{-1} \iint_{D_{2}}\left(|\mathbf{U}|^{2}-h_{2}\right)_{+}^{(s+1) / 2} d x d \tau .
\end{array}
$$

Proof. For the sake of simplicity, assume that $\mathbf{U}$ is the strong solution of (1), so that it is possible to work pointwise. Assume for the moment $s \geqslant 1$.

Let $\zeta=\zeta(\tau)$ be a smooth cutoff function in $(0, t)$, such that $\zeta=1$ for $t_{1} \leqslant \tau \leqslant t, \zeta=0$ out of $0<\tau \leqslant t_{2},\left|\zeta_{\tau}\right| \leqslant c\left(t_{1}-t_{2}\right)^{-1}$.

Multiply both sides of (1) by $u_{i}\left(|\mathbf{U}|^{2}-h_{2}\right)_{+}^{\frac{s-1}{2}} \zeta^{p}(\tau), i=1, . ., l$, integrate by parts and sum with the respect the index $i$ to get

$$
\begin{aligned}
& \frac{1}{s+1} \int_{\mathbb{R}^{N}} \zeta^{p}\left(|\mathbf{U}|^{2}-h_{2}\right)_{+}^{\frac{s+1}{2}} d x \\
&+\sum_{i=1}^{l} \iint_{D_{2}} \zeta^{p}\left(|\mathbf{U}|^{m-1}|\nabla \mathbf{U}|^{p-2} \nabla u_{i}\right) \nabla\left(u_{i}\left(|\mathbf{U}|^{2}-h_{2}\right)_{+}^{\frac{s-1}{2}}\right) d x d \tau \\
&=-\frac{p}{s+1} \iint_{D_{2}} \zeta^{p-1} \zeta_{\tau}\left(|\mathbf{U}|^{2}-h_{2}\right)_{+}^{\frac{s+1}{2}} d x d \tau .
\end{aligned}
$$


We have

$$
\begin{gathered}
\sum_{i=1}^{l}\left(|\mathbf{U}|^{m-1}|\nabla \mathbf{U}|^{p-2} \nabla u_{i}\right) \nabla\left(u_{i}\left(|\mathbf{U}|^{2}-h_{2}\right)_{+}^{\frac{s-1}{2}}\right) \\
=|\mathbf{U}|^{m-1}|\nabla \mathbf{U}|^{p}\left(|\mathbf{U}|^{2}-h_{2}\right)_{+}^{\frac{s-1}{2}} \\
\quad+\frac{s-1}{4}|\mathbf{U}|^{m-1}|\nabla \mathbf{U}|^{p-2}\left(\nabla|\mathbf{U}|^{2}\right)^{2}\left(|\mathbf{U}|^{2}-h_{2}\right)_{+}^{\frac{s-1}{2}-1} \\
\geqslant|\mathbf{U}|^{m-1}|\nabla \mathbf{U}|^{p}\left(|\mathbf{U}|^{2}-h_{2}\right)_{+}^{\frac{s-1}{2}} .
\end{gathered}
$$

Notice that when $|\mathbf{U}|^{2}>h_{1}$ we have

$$
\begin{aligned}
|\mathbf{U}|^{2} & =|\mathbf{U}|^{2}-h_{2}+h_{2} \frac{h_{1}-h_{2}}{h_{1}-h_{2}} \\
& \leqslant\left(|\mathbf{U}|^{2}-h_{2}\right)+h_{2} \frac{|\mathbf{U}|^{2}-h_{2}}{h_{1}-h_{2}}=C\left(h_{1}, h_{2}\right)\left(|\mathbf{U}|^{2}-h_{2}\right) .
\end{aligned}
$$

Moreover,

$$
\left|\nabla\left(|\mathbf{U}|^{2}-h_{1}\right)\right|^{p}=|\nabla(|\mathbf{U}|)|^{p} 2^{p}|\mathbf{U}|^{p} .
$$

Noting that

$$
|\mathbf{U}|^{m-1}|\nabla \mathbf{U}|^{p}\left(|\mathbf{U}|^{2}-h_{2}\right)_{+}^{\frac{s-1}{2}} \geqslant|\mathbf{U}|^{m-1}|\nabla| \mathbf{U}||^{p}\left(|\mathbf{U}|^{2}-h_{2}\right)_{+}^{\frac{s-1}{2}}
$$

by (18) we have

$$
|\mathbf{U}|^{m-1}|\nabla \mathbf{U}|^{p}\left(|\mathbf{U}|^{2}-h_{2}\right)_{+}^{\frac{s-1}{2}} \geqslant 2^{-p}\left(|\mathbf{U}|^{2}-h_{2}\right)_{+}^{\frac{s-1}{2}}|\mathbf{U}|^{m-1-p}\left|\nabla\left(|\mathbf{U}|^{2}-h_{1}\right)\right|^{p} .
$$

Therefore, if $m-1-p<0$ by (17),

$$
\begin{aligned}
|\mathbf{U}|^{m-1}|\nabla \mathbf{U}|^{p}\left(|\mathbf{U}|^{2}-h_{2}\right)_{+}^{\frac{s-1}{2}} & \\
& \geqslant 2^{-p} C\left(h_{1}, h_{2}\right)^{-\frac{m-1-p}{2}}\left(|\mathbf{U}|^{2}-h_{1}\right)_{+}^{\frac{s-2+m-p}{2}}\left|\nabla\left(|\mathbf{U}|^{2}-h_{1}\right)\right|^{p}
\end{aligned}
$$

If $m-1-p \geqslant 0$ we have that $|\mathbf{U}|^{m-1-p} \geqslant\left|\left(\mathbf{U}-h_{1}\right)_{+}\right|^{m-1-p}$ and again we can prove (19). Combining (16), (19), and by the definition of the cut-off $\zeta$, we get (15). If $-1<s<1$, for each $n \in N$ consider the test function

$$
\psi_{n}=u_{i}\left[\left(|\mathbf{U}|^{2}-h_{2}\right)_{+}^{\frac{s-1}{2}} \wedge n\right] \zeta^{p}(\tau)
$$

Letting $n \rightarrow \infty$ one can get (15).

REMARK 2 Note that the proof of Lemma 3 works also in the more general case of equation (8).

In the sequel we need an interpolation inequality and the celebrated Gagliardo-Nirenberg inequality (for both these results, see, for instance, Chapter 0 of [10]). 
LEMMA 4 Let $\left\{Y_{n}\right\}_{n=1}^{\infty}$ be a sequence of equi-bounded positive numbers satisfying the recursive inequalities

$$
Y_{n} \leqslant C b^{n} Y_{n+1}^{1-\alpha}
$$

where $C, b>1$ and $\alpha \in(0,1)$ are given constants. Then

$$
Y_{0} \leqslant\left(2 C b^{\frac{1-\alpha}{\alpha}}\right)^{\frac{1}{\alpha}}
$$

LEMma 5 (see also [18], Chapter 2, Section 5) Let $\left\{Y_{n}\right\}_{n=1}^{\infty}$ be a sequence of equi-bounded positive numbers satisfying the recursive inequalities

$$
Y_{n} \leqslant C b^{n} Y_{n+1}^{1+\alpha}
$$

where $C, b>1$ and $\alpha>0$ ) are given constants. Then, if

$$
Y_{0} \leqslant C^{-\frac{1}{\alpha}} b^{-\frac{1}{\alpha^{2}}}
$$

then $Y_{n} \rightarrow 0$ when $n \rightarrow+\infty$.

LEMMA 6 Let $u \in W^{1, p}\left(\mathbb{R}^{N}\right)$ with $p>1$. Let $0<\mu<q<\frac{p N}{N-p}$ if $p<N$ and $0<\mu<q$ otherwise

$$
\|u\|_{q} \leqslant C\|D u\|_{p}^{\alpha}\|u\|_{\mu}^{1-\alpha}
$$

where $C$ is a constant depending only by $N, p, q, \mu$ and

$$
\alpha=\left(\frac{1}{\mu}-\frac{1}{q}\right)\left(\frac{1}{N}-\frac{1}{p}+\frac{1}{\mu}\right)^{-1} .
$$

Note that in general the Gagliardo-Nirenberg inequality is stated with the assumption that $1<$ $\mu<q$. However, the proof works also in the weaker assumption $0<\mu<q$, even if, in general, the space $L^{\mu}\left(\mathbb{R}^{N}\right)$ and $L^{q}\left(\mathbb{R}^{N}\right)$ are no longer Banach spaces.

\section{Proof of Theorem 1}

The idea is to prove that there exists a $p>1$ such that $\left(|\mathbf{U}|^{2}-a\right)_{+}^{\frac{1}{2}} \in L^{p}\left(\mathbb{R}^{N}\right)$, where $a$ is a suitable positive constant. Then, by applying De Giorgi iterative scheme, starting from this estimate, we are able to prove that $|\mathbf{U}|$ is bounded.

Consider two positive times $0<\tau_{2}<\tau_{1}$ and two positive constants $0<a_{2}<a_{1}$. Let $\vartheta_{n}=$ $\tau_{2}+\left(\tau_{1}-\tau_{2}\right) 2^{-n}, k_{n}=a_{2}+\left(a_{1}-a_{2}\right) 2^{-n}, n \geqslant 0$. Apply Lemma 3 with $t_{1}=\vartheta_{n}, t_{2}=\vartheta_{n+1}$; $h_{1}=k_{n}, h_{2}=k_{n+1}$. Choose $s>0$ such that $s+m-p-2>0$.

Notice that

$$
\begin{aligned}
& \left|\nabla\left(|\mathbf{U}|^{2}-k_{2 n+1}\right)_{+}^{\frac{s+m+p-2}{2 p}}\right|^{p} \\
& \quad=\left(\frac{s+m+p-2}{p}\right)^{p}\left(|\mathbf{U}|^{2}-k_{2 n+1}\right)_{+}^{\frac{s+m-2-p}{2}}\left|\nabla\left(|\mathbf{U}|^{2}-k_{2 n+1}\right)_{+}\right|^{p} .
\end{aligned}
$$


Then, we have

$$
\begin{aligned}
& \sup _{\vartheta_{2 n}<\tau<t} \int_{\mathbb{R}^{N}}\left(|\mathbf{U}|^{2}-k_{2 n+1}\right)_{+}^{\frac{s+1}{2}} d x+\iint_{S_{2 n+1}}\left|\nabla\left(|\mathbf{U}|^{2}-k_{2 n+1}\right)_{+}^{\frac{s+m+p-2}{2 p}}\right|^{p} d x d \tau \\
& \leqslant c 2^{n}\left(\tau_{1}-\tau_{2}\right)^{-1} \iint_{S_{2 n+2}}\left(|\mathbf{U}|^{2}-k_{2 n+2}\right)_{+}^{\frac{s+1}{2}} d x d \tau
\end{aligned}
$$

where $S_{n}=\mathbb{R}^{N} \times\left(\vartheta_{n}, t\right)$. Let $z_{n}$ be a smooth cut-off function such that $0 \leqslant z_{n} \leqslant 1, z_{n}=1$ in $S_{2 n}, z_{n}=0$ outside of $S_{2 n+1}, 0 \leqslant z_{n \tau} \leqslant 2^{2 n+1}\left(\tau_{1}-\tau_{2}\right)^{-1}$. We have

$$
\iint_{S_{2 n+1}}\left|\nabla\left(|\mathbf{U}|^{2}-k_{2 n}\right)_{+}^{\frac{s+m+p-2}{2 p}} z_{n}\right|^{p} d x d \tau \leqslant \iint_{S_{2 n+1}}\left|\nabla\left(|\mathbf{U}|^{2}-k_{2 n+1}\right)_{+}^{\frac{s+m+p-2}{2 p}}\right|^{p} d x d \tau .
$$

Thus, defining

$$
v_{n}=\left(|\mathbf{U}|^{2}-k_{2 n}\right)_{+}^{\frac{s+m+p-2}{2 p}} z_{n}
$$

from (21) with $q=\frac{p(s+1)}{s+m+p-2}$ we get

$$
\sup _{\vartheta_{2 n}<\tau<t} \int_{\mathbb{R}^{N}} v_{n}^{q} d x+\iint_{S_{2 n+1}}\left|\nabla v_{n}\right|^{p} d x d \tau \leqslant c 2^{2 n}\left(\tau_{1}-\tau_{2}\right)^{-1} \iint_{S_{2 n+2}} v_{n+1}^{q} d x d \tau .
$$

Note that if $m+p>3$ we have $q<p$ and therefore we are in the degenerate case. Moreover if the Barenblatt exponent $\lambda=N(m+p-3)+p$ is positive, we can use the Gagliardo-Nirenberg inequality (see Lemma 6). Therefore,

$$
\int_{\mathbb{R}^{N}} v_{n+1}^{q} d x \leqslant c\left(\int_{\mathbb{R}^{N}}\left|\nabla v_{n+1}\right|^{p} d x\right)^{\alpha q / p}\left(\int_{\mathbb{R}^{N}} v_{n+1}^{\mu} d x\right)^{(1-\alpha) q / \mu},
$$

where $\mu=\frac{p}{s+m-2+p}$.

Define

$$
A:=\frac{\alpha q}{p}, \quad B:=\frac{(1-\alpha) q}{\mu} .
$$

Note that $A<1$ if $\lambda=N(m+p-3)+p>0$.

Integrating in time and applying Young's inequalities to (23), we obtain

$$
\iint_{S_{2 n+2}} v_{n+1}^{q} d x d \tau \leqslant\left(\iint_{S_{2 n+2}}\left|\nabla v_{n+1}\right|^{p} d x d \tau\right)^{A}\left(\int_{\vartheta_{2 n+2}}^{t}\left(\int_{\mathbb{R}^{N}} v_{n+1}^{\mu} d x\right)^{\frac{B}{1-A}} d \tau\right)^{1-A} .
$$

Therefore, we get

$$
\iint_{S_{2 n+2}} v_{n+1}^{q} d x d \tau \leqslant c\left(t-\vartheta_{2 n+2}\right)^{1-A}\left(\iint_{S_{2 n+2}}\left|\nabla v_{n+1}\right|^{p} d x d \tau\right)^{A} \sup _{\vartheta_{2 n+2}<\tau<t}\left(\int_{\mathbb{R}^{N}} v_{n+1}^{\mu}(\tau) d x\right)^{B} .
$$


Note that by Lemma 2

$$
\sup _{\vartheta_{2 n+2}<\tau<t} \int_{\mathbb{R}^{N}} v_{n+1}^{\mu}(\tau) d x \leqslant \int_{\mathbb{R}^{N}}\left|\mathbf{U}_{0}\right| d x
$$

so the quantity on the right in $(25)$ is finite.

Combining (22) and (25), we get

$$
\begin{aligned}
J_{n} & :=\sup _{\vartheta_{2 n}<\tau<t} \int_{\mathbb{R}^{N}} v_{n}^{q} d x+\iint_{S_{2 n+1}}\left|\nabla v_{n}\right|^{p} d x d \tau \\
& \leqslant b_{1}^{n}\left(\tau_{1}-\tau_{2}\right)^{-1}\left(t-\vartheta_{2 n+2}\right)^{1-A}\left(\sup _{\vartheta_{2 n+2}<\tau<t} \int v_{\mathbb{R}^{N}}^{\mu}(\tau) d x\right)^{B} J_{n+1}^{A}
\end{aligned}
$$

where $b_{1}$ a constant greater than 1 .

Thus, by the iterative Lemma 4 we get

$$
\sup _{\tau_{1}<\tau<t} \int_{\mathbb{R}^{N}}\left(|\mathbf{U}|^{2}-a_{2}\right)_{+}^{\frac{s+1}{2}} d x \leqslant c\left(\tau_{1}-\tau_{2}\right)^{-\frac{1}{1-A}}\left(t-\tau_{2}\right)\left(\sup _{\tau_{2}<\tau<t} \int_{\mathbb{R}^{N}}\left(|\mathbf{U}|^{2}-a_{1}\right)_{+}^{\frac{1}{2}} d x\right)^{\frac{B}{1-A}}
$$

and this implies the higher integrability of $\left(|\mathbf{U}|^{2}-a_{2}\right)_{+}^{\frac{1}{2}}$.

To prove the boundedness of $|\mathbf{U}|$ we have to apply a DeGiorgi iterative scheme.

Let $t_{j}=t / 2\left(1-2^{-j}\right), k_{j}=k\left(1-2^{-j-1}\right)$, and $\bar{k}_{j}=\left(k_{j}+k_{j+1}\right) / 2$.

Then, in (27) plug $\tau_{1}=t_{j+1}, \tau_{2}=t_{j}, a_{2}=\bar{k}_{j}, a_{1}=k_{j}$, to get

$$
\sup _{t_{j+1}<\tau<t} \int_{\mathbb{R}^{N}}\left(|\mathbf{U}|^{2}-\bar{k}_{j}\right)_{+}^{\frac{s+1}{2}} d x \leqslant c b_{2}^{j} t^{-\frac{A}{1-A}}\left(\sup _{t_{j}<\tau<t} \int_{\mathbb{R}^{N}}\left(|\mathbf{U}|^{2}-k_{j}\right)_{+}^{1 / 2} d x\right)^{1+\frac{B+A-1}{1-A}} .
$$

Note that, as $s>0$ and $\lambda=N(m+p-3)+p>0$, we have $B+A>1$.

Denote

$$
M_{j+1}=\sup _{t_{j+1}<\tau<t} \int_{\mathbb{R}^{N}}\left(|\mathbf{U}|^{2}-k_{j+1}\right)_{+}^{1 / 2} d x .
$$

Since

$$
\int_{\mathbb{R}^{N}}\left(|\mathbf{U}|^{2}-\bar{k}_{j}\right)_{+}^{\frac{s+1}{2}} d x \geqslant\left(k_{j+1}-\bar{k}_{j}\right)^{s} \int_{\mathbb{R}^{N}}\left(|\mathbf{U}|^{2}-k_{j+1}\right)_{+}^{1 / 2} d x
$$

from (28) one gets

$$
M_{j+1} \leqslant c b_{3}^{j} t^{-\frac{A}{1-A}} k^{-s} M_{j}^{1+\frac{A+B-1}{1-A}} .
$$

Noting that $\frac{N s}{\lambda}=\frac{A}{1-A}$ and $\frac{p s}{\lambda}=\frac{A+B-1}{1-A}$, we have that

$$
M_{j+1} \leqslant c b_{3}^{j} t^{-\frac{N s}{\lambda}} k^{-s} M_{j}^{1+\frac{p s}{\lambda}}
$$


It follows from Lemma 5 that $M_{j} \rightarrow 0$ as $j \rightarrow \infty$ if

$$
k=C t^{-\frac{N}{\lambda}} \widetilde{M}_{0}^{\frac{p}{\lambda}}
$$

where $\widetilde{M}_{0}(t)=\sup _{t / 4<\tau<t} \int_{\mathbb{R}^{N}}|\mathbf{U}(t, x)| d x$.

Therefore, by Lemma 2, (29) holds true if

$$
k=C t^{-\frac{N}{\lambda}}\left(\int_{\mathbb{R}^{N}}\left|\mathbf{U}_{\mathbf{0}}(x)\right| d x\right)^{\frac{p}{\lambda}},
$$

which implies (6)

REMARK 3 Note that the proof of Theorem 1 works exactly in the more general case of equation (8).

\section{Proof of Theorem 2}

At the beginning, the proof is similar to the one of Lemma 3. Consider the sequence $r_{n}=2 r(1-$ $\left.2^{-n-1}\right), n=0,1, \ldots, r>2 R_{0}, \bar{r}_{n}=\frac{\left(r_{n}+r_{n+1}\right)}{2}, \Omega_{n}=R^{N} \backslash B_{r_{n}}(0), \bar{\Omega}_{n}=R^{N} \backslash B_{\bar{r}_{n}}(0)$.

Let $\eta_{n}(x)$ be a sequence of cutoff functions satisfying:

$$
\eta_{n}(x)=0 \text { for } x \in B_{r_{n}}(0), \eta_{n}(x)=1 \text { for } x \in \bar{\Omega}_{n},\left|\nabla \eta_{n}\right| \leqslant c 2^{n} r^{-1} .
$$

Let $\theta$ a positive constant that will be fixed later. Then, multiplying both sides of (1) by $\eta_{n}^{p}|\mathbf{U}|^{\theta-1} u_{j}$, $i=1, \ldots, l$ and assume $\theta \geqslant 1$. By integrating over $S_{t}$, we obtain

$$
\begin{aligned}
& \frac{1}{\theta+1} \int_{\mathbb{R}^{N}} \eta_{n}^{p}|\mathbf{U}|^{\theta+1} d x \\
& +\iint_{S_{t}} \eta_{n}^{p}|\mathbf{U}|^{m-1}|\nabla \mathbf{U}|^{p-2} \nabla u_{j} \nabla\left(|\mathbf{U}|^{\theta-1} u_{j}\right) d x d \tau \\
& =-p \iint_{S_{t}} \eta_{n}^{p-1}|\mathbf{U}|^{m-1}|\nabla \mathbf{U}|^{p-2} \nabla u_{j} \nabla \eta_{n}|\mathbf{U}|^{\theta-1} u_{j} d x d \tau .
\end{aligned}
$$

Noting that

$$
\begin{aligned}
& |\nabla \mathbf{U}|^{p-2} \nabla u_{j} \nabla\left(|\mathbf{U}|^{\theta-1} u_{j}\right)=|\mathbf{U}|^{\theta-1}|\nabla \mathbf{U}|^{p-2}\left(|\nabla \mathbf{U}|^{2}+(\theta-1)|\nabla| \mathbf{U}||^{2}\right) \\
& \geqslant \theta|\mathbf{U}|^{\theta-1}|\nabla \mathbf{U}|^{p} .
\end{aligned}
$$

and that, by the Cauchy inequality,

$$
\begin{aligned}
& \eta_{n}^{p-1}|\mathbf{U}|^{m-1}|\nabla \mathbf{U}|^{p-2} \nabla u_{j} \nabla \eta_{n}|\mathbf{U}|^{\theta-1} u_{j} \\
& \leqslant \frac{\theta}{2 p} \eta_{n}^{p}|\mathbf{U}|^{m+\theta-2}|\nabla \mathbf{U}|^{p}+\gamma\left|\nabla \eta_{n}\right|^{p}|\mathbf{U}|^{p+m+\theta-2}
\end{aligned}
$$


estimate (30) yields

$$
\begin{aligned}
& \sup _{0<\tau<t} \int_{\bar{\Omega}_{n}}|\mathbf{U}|^{\theta+1} d x+\int_{0}^{t} \int_{\bar{\Omega}_{n}} \eta_{n}^{p}|\mathbf{U}|^{m+\theta-2}|\nabla \mathbf{U}|^{p} d x d \tau \\
& \leqslant c \frac{2^{p n}}{r^{p}} \int_{0}^{t} \int_{\Omega_{n} \backslash \bar{\Omega}_{n}}|\mathbf{U}|^{p+m+\theta-2} d x d \tau .
\end{aligned}
$$

If $0<\theta<1$ for each $m \in \mathbb{N}$, consider $\eta_{n}^{p}\left(|\mathbf{U}|^{\theta-1} \wedge m\right) u_{j}$, repeat the previous argument and pass to the limit when $m \rightarrow \infty$ to get again (31).

Let $\zeta_{n}(x)$ be such that $\zeta_{n}=0$ for $x \in \bar{\Omega}_{n}$ and $\zeta_{n}=1$ for $x \in \Omega_{n+1}$. Let $v_{n}=$ $|\mathbf{U}|^{(p+m+\theta-2) / p} \zeta_{n}^{s}$. Then, from (31), we get

$$
\sup _{0<\tau<t} \int_{\mathbb{R}^{N}} v_{n}^{q} d x+\int_{0}^{t} \int_{\mathbb{R}^{N}}\left|\nabla v_{n}\right|^{p} d x d \tau \leqslant c \frac{2^{2 n}}{r^{p}} \int_{0}^{t} \int_{\mathbb{R}^{N}} v_{n}^{p} d x d \tau
$$

with $q=\frac{p(1+\theta)}{p+m+\theta-2}$. Note that if $m+p>3$ then $q<p$.

Using the Gagliardo-Nirenberg inequality, we get

$$
\int_{\mathbb{R}^{N}} v_{n-1}^{p} d x \leqslant c\left(\int_{\mathbb{R}^{N}}\left|\nabla v_{n-1}\right|^{p} d x\right)^{\alpha}\left(\int_{\mathbb{R}^{N}} v_{n-1}^{q} d x\right)^{p(1-\alpha) / q}
$$

with

$$
\alpha=\frac{N(p+m-3)}{N(p+m-3)+p(1+\theta)} .
$$

By integrating the previous inequality in time, we obtain

$$
\int_{0}^{t} \int_{\mathbb{R}^{N}} v_{n-1}^{p} d x d \tau \leqslant c t^{1-\alpha}\left(\int_{0}^{t} \int_{\mathbb{R}^{N}}\left|\nabla v_{n-1}\right|^{p} d x d \tau\right)^{\alpha} \times\left(\sup _{0<\tau<t} \int_{\mathbb{R}^{N}} v_{n-1}^{q}(\tau, x) d x\right)^{p(1-\alpha) / q} .
$$

Therefore, combining (32) and (34), we get

$$
I_{n}=\sup _{0<\tau<t} \int_{\mathbb{R}^{N}} v_{n}^{q} d x+\int_{0}^{t} \int_{\mathbb{R}^{N}}\left|\nabla v_{n-1}\right|^{p} d x d \tau \leqslant \gamma \frac{b^{n}}{r^{p}} t^{1-\alpha} I_{n-1}^{1+(1-\alpha)\left(\frac{p}{q}-1\right)} .
$$

Then, by iterative Lemma 5, we conclude that $I_{n} \rightarrow 0$ as $n \rightarrow \infty$ provided

$$
\frac{t^{1-\alpha}}{r^{p}} I_{0}^{(1-\alpha)\left(\frac{p}{q}-1\right)} \leqslant \varepsilon
$$

where $\varepsilon=\varepsilon(m, N, \theta)$ is small enough. Notice that by (32) we have

$$
I_{0} \leqslant \frac{c}{r^{p}} \int_{0}^{t} \int_{\mathbb{R}^{N}}|\mathbf{U}|^{p+m+\theta-2} d x d \tau \leqslant \frac{c}{r^{p}} \int_{0}^{t}\left(\||\mathbb{U}(\tau)|\|_{\infty}^{p+m+\theta-3} \int_{\mathbb{R}^{N}}|\mathbf{U}(\tau, x)| d x\right) d \tau .
$$


By (11)

$$
I_{0} \leqslant \frac{c}{r^{p}}\left\|\left|\mathbf{U}_{0}\right|\right\|_{1} \int_{0}^{t}\||\mathbb{U}(\tau)|\|_{\infty}^{p+m+\theta-3} d \tau .
$$

Choose $\theta$ so small that

$$
0<1-\frac{N(p+m+\theta-3)}{\lambda}
$$

By (6) and (36)

$$
\begin{aligned}
I_{0} & \leqslant \frac{\gamma}{r^{p}}\left\|\left|\mathbf{U}_{0}\right|\right\|_{1}^{1+\frac{p(p+m+\theta-3)}{\lambda}} \int_{0}^{t} \tau^{-\frac{N(p+m+\theta-3)}{\lambda}} d \tau \\
& =\frac{\gamma t^{1-\frac{N(p+m+\theta-3)}{\lambda}}}{r^{p}}\left\|\left|\mathbf{U}_{0}\right|\right\|_{1}^{1+\frac{p(p+m+\theta-3)}{\lambda}} .
\end{aligned}
$$

Plugging now (37) in (35) and recalling (33), we deduce that $|\mathbf{U}|=0$ outside of $|x| \leqslant 4 R_{0}+$ $c\left\|\left|\mathbf{U}_{0}\right|\right\|_{1}^{\frac{p+m-3}{\lambda}} t^{\frac{1}{\lambda}}$.

REMARK 4 Note that the proof of Theorem 2 works exactly in the more general case of equation (8).

Acknowledgement. The authors have been supported by the Gruppo Nazionale per l'Analisi Matematica, la Probabilità e le loro Applicazioni (GNAMPA) of the Istituto Nazionale di Alta Matematica (INdAM). We also want to thank the anonymous referees for their valuable suggestions.

\section{REFERENCES}

1. Alt, H. W. \& Luckhaus, S., Quasilinear elliptic-parabolic differential equations. Math. Z. 183 (1983), 311-341. Zb10497.35049 MR0706391

2. Andreucci, D. \& Tedeev, A. F., Sharp estimates and finite speed of propagation for a Neumann problem in domains narrowing at infinity. Adv. Diff. Eqns 5 (2000), 833-860. Zb10987. 35090 MR1776343

3. Andreucci, D. \& TedeEv, A. F., Finite speed of propagation for thin film equations and other higher order parabolic equations with general nonlinearity, Interfaces Free Bound. 3 (2001), 233-264. Zbl1002 . 35059 MR1843587

4. Andreucci, D. \& Tedeev, A. F., Universal bounds at the blow-up time for nonlinear parabolic equations, Adv. Diff. Eqns 10 (2005), 89-120. Zb11122. 35042 MR2106122

5. A. Anile, M., Allegretto, W. \& Ringhofer, C., Mathematical Problems in Semiconductor Physics. Lecture Notes in Mathematics 1823, Lectures given at the C.I.M.E, Summer School, Editor: A. M. Anile (2003). MR2070860

6. Antontsev, S., Diaz, J. I. \& Shmarev, S., Energy Methods for Free Boundary Problems : Applications to Nonlinear, in "PDEs and Fluid Mechanics". Birkhauser, Boston, Progress in Nonlinear Differential Equations and Their Applications, 48 (2002). Zb101682098 MR1858749

7. BERNIS, F., Existence results for doubly nonlinear higher order parabolic equations on unbounded domain. Math. Ann., 279 (1988), 373-393. Zb10609. 35048 MR0922422

8. DiBenedetto, E., Degenerate Parabolic Equations. Universitext, Springer Verlag (1993). Zb10794 35090 MR1230384

9. DiBenedetto E. \& Friedman, A., Hölder estimates for nonlinear parabolic systems. J. Reine Ang. Math. 357 (1985), 1-22. Zb10549. 35061 MR0783531 
10. DiBenedetto, E., Gianazza, U. \& Vespri, V., Harnack's inequality for degenerate and singular parabolic equations. Springer Monographs in Mathematics, Springer Verlag (2012). Zbl1237. 35004 MR2865434

11. Fornaro, S. \& Sosio, M., Intrinsic Harnack estimates for some doubly nonlinear degenerate parabolic equations. Adv. Diff. Equs. 13 (2008), 139-168. Zbl1160. 35039 MR2482539

12. IsHige, K., On the existence of solutions of the Cauchy problem for a doubly nonlinear parabolic equations. SIAM J. Math. Anal., 7 (1996), 1235-1260. Zb10858. 35056 MR1402438

13. IVANov, A. V., Hölder estimates for quasilinear doubly degenerate parabolic equations. J. Soviet Mathematics 56 (1991), 2320-2347. Zbl0729.35018 MR1031986

14. IVAnov, A. V., Regularity for doubly nonlinear parabolic equations. Zap. Nauchn. Sem. S.-Peterburg. Otdel. Mat. Inst. Steklov (POMI) 209 (1994), 37-94. Zb10856. 35065 MR1328634

15. Kalashnikov, A.S., Some properties of the qualitative theory of nonlinear degenerate second-order parabolic equations. Russian Math. Surveys 42 (1987), 169-222. Zbl0642.35047

16. JÜngel, A., Transport Equations for Semiconductors. Lectures Notes in Physics 773. Springer (2009). MR2723324

17. Jüngel, A. , Markovich, P. A. \& Toscani, G., Decay rates for solutions of degenerate parabolic systems. USA-Chile Workshop on Nonlinear Analysis, Electron. J. Diff. Eqns., Conf. 06 (2001), 189-202. Zb10964.35085 MR1804774

18. Ladyzhenskaya, O. A., Solonnikov, V. A. \& Ural'ceva, N. N., Linear and Quasilinear Equations of Parabolic Type. Volume 23 of Translation of Mathematical Monograghs, American Mathematical Society, Providence, RI (1968). Zb10174. 15403 MR0241822

19. Lions, J. L., Quelques méthodes de résolution des problèmes aux limites non linéaires. Dunod, Paris (1969). Zbl0189.40603 MR0259693

20. Porzio, M. M. \& VESPRI, V., Hölder estimates for local solutions of some doubly nonlinear degenerate parabolic equations. J. Diff. Eqns. 10 (1993), 146-178. Zb10796. 35089 MR1218742

21. Trudinger, N. S., Pointwise estimates and quasilinear parabolic equations. Comm. Pure Appl. Math. 21 (1968), 205-226. Zbl0159. 39303 MR0226168

22. Tsutsumi, M., On solutions of some doubly nonlinear degenerate parabolic equations with absorption. J. Math. Anal. Appl. 132 (1988), 187-212. Zbl0681. 35047 MR0942364

23. VÁzquez, J. L., The Porous Medium Equation, Mathematical Theory. Oxford Mathematical Monographs, (2007).

24. VESPRI, V., Harnack type inequalities for solutions of certain doubly nonlinear parabolic equations. $J$. Math. Anal. Appl. 181 (1994 ), 104-131. Zb10798.35073 MR1257957

25. WAng, S., Doubly Nonlinear Degenerate Parabolic Systems with Coupled Nonlinear Boundary Conditions. J. Diff. Eqns. 182 (2002), 431-469. Zbl1005. 35053 MR1900330

26. On $p$-Laplacian type of evolution system and applications to the Bean model in the type-II superconductivity theory. Quart. Appl. Math. 59 (2001), 47-66. Zbl1030. 35109 MR0807.35076

27. YIN, H. M., A degenerate evolution system modelling Bean's critical-state type-II superconductors. Discrete Cont. Dynamical Systems 8 (2002), 781-794. Zbl1006. 35091 MR1897881

28. Yin, H. M., On a degenerate parabolic system. J. Diff. Eqns. 245 (2008), 722-736. Zbl1157. 35062 MR2422525

29. YUAN, H., The Cauchy problem for a quasilinear degenerate parabolic system. Nonlinear Anal. 23 (1994), 155-164. Zb10807.35076 MR1289124 